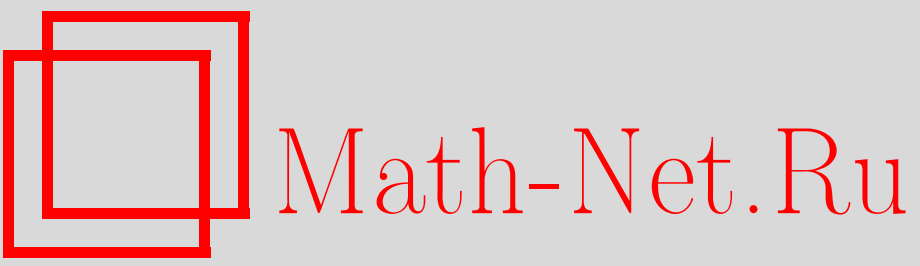

В. Е. Зобов, М. А. Попов, О координате особой точки временной корреляционной функции спиновой системы на простой гиперкубической решетке при высоких температурах, ТMФ, 2002, том 131, номер 3, 491-502

DOI: https://doi.org/10.4213/tmf342

Использование Общероссийского математического портала Math-Net.Ru подразумевает, что вы прочитали и согласны с пользовательским соглашением

http://www.mathnet.ru/rus/agreement

Параметры загрузки:

IP : 34.229 .108 .108

26 апреля 2023 г., 14:54:05 


\title{
О КООРДИНАТЕ ОСОБОЙ ТОЧКИ ВРЕМЕННОЙ КОРРЕЛЯЦИОННОЙ ФУНКЦИИ СПИНОВОЙ СИСТЕМЫ НА ПРОСТОЙ ГИПЕРКУБИЧЕСКОЙ РЕШЕТКЕ ПРИ ВЫСОКИХ ТЕМПЕРАТУРАХ
}

\begin{abstract}
Методом разложения по обратной размерности пространства $d^{-1}$ получена оценка координаты особой точки на оси мнимого времени у зависящей от времени автокорреляционной функции гейзенберговской модели со спином $1 / 2$ на простой гиперкубической решетке при высоких температурах. Коэффициенты ряда по степеням времени (спектральные моменты) автокорреляционной функции представлены в виде сумм нагруженных решеточных фигур, в которых деревья, построенные из двойных связей, дают главные вклады по $d^{-1}$, а такие же деревья с встроенными в них квадратами из шести связей или диаграммами с четырехкратной связью дают вклады следующего порядка малости. От этих последних вкладов найдены поправки к координате особой точки автокорреляционной функции.
\end{abstract}

\section{1. ВВЕДЕНИЕ}

В высокотемпературной динамике спиновых систем, как и в других системах многих тел, важную роль играют многочастичные эффекты. Они определяют скорость установления равновесия и проявляются, например, в форме "крыльев" спектров корреляционных функций. Наблюдение "крыльев" спектров корреляционных функций ядерных спинов в твердых телах, выполненное методами магнитного резонанса, выявило, что их форма в ряде случаев описывается экспоненциальной функцией вместо ожидаемой функции Гаусса [1]-[6]. Если это так, то у корреляционных функций должны быть особые точки на оси мнимого времени,которые могут указывать на новый тип коллективных эффектов в таких системах. Низкая точность регистрации слабого сигнала на "крыле" спектра не позволяет сделать однозначный вывод о его форме.

Теоретические исследования [7]-[9] подтвердили возможность сушествования у корреляционных функций жестких спиновых решеток при высоких температурах особых

${ }^{*}$ Институт физики им. Л.В. Киренского СО РАН, Красноярск, Россия. E-mail: rsa@iph.krasn.ru,root@iph.krasnoyarsk.su

${ }^{\dagger}$ Красноярский государственный университет, Красноярск, Россия 
точек на оси мнимого времени, по крайней мере, для решеток большой размерности $d$. Этому выводу не противоречат и результаты компютерного моделирования [10]-[12] для двух- и трехмерных решеток. Причина изменения формы "крыла" спектра объясняется флуктуациями во времени локального магнитного поля на спине, происходящими вследствие переворотов соседних спинов, создаюших это поле. В свою очередь, причиной переворотов служит все то же внутреннее взаимодействие между спинами (диполь-дипольное или обменное). Координату особой точки удается достаточно легко рассчитать в приближении самосогласованного флуктуируюшего локального поля для решеток большой размерности [7]-[9], [13], [14]. Если представить коэффишиенты ряда по степеням времени (спектральные моменты) спиновой корреляционной функции в виде суммы нагруженных решеточных фигур [15], то в этом приближении в сумме остаются деревья, построенные из двойных связей на решетке Бете [7]-[12], [14], т.е. без какого-либо взаимного влияния ветвей, кроме ограничения на максимальное число ветвей $Z=2 d$, выходящих из узла. Весовой множитель определяется числом способов построения данного дерева, выполняемого по определенным правилам: первые связи присоединяются последовательно от корня к концу веток, а вторые - последовательно от конца веток к корню.

В спиновых системах на гиперкубических решетках ветви могут пересекаться. Однако вклад петли из связей, образуюшейся при простом наложении узлов разных ветвей, не совпадает с вкладом аналогичной петли, содержащейся в точных коэффициентах временно́го ряда [15], поскольку правила их построения многократными коммутациями гамильтониана при заданной последовательности связей сложнее. Поэтому для исправления результата приближения самосогласованного флуктуируюшего локального поля (приближения Бете) следует первый вклад заменить на второй. Вследствие такой замены изменятся величины моментов и координаты особой точки.

В теории многих тел для расчетов таких характеристик, как температура фазовых переходов, параметры роста кластеров ( "lattice animals", линейных и разветвленных полимеров), являюшихся величинами, обратными к координатам особых точек производящих функций, разработан метод разложения по обратной размерности пространства (см., например, [16], [17]). Установлено, что уже при $d=2$ и $d=3$ этот метод дает искомые значения с точностью около $5 \%$ и $1 \%$, соответственно. Поскольку кластеры в высокотемпературной спиновой динамике отличаются свойствами от кластеров, изученных в работах [16], [17], то полученные формулы к ним непосредственно неприменимы.

В работе [9] метод разложения по $d^{-1}$ был обобщен на деревья, построенные из двойных связей, а также учтен эффект исключения объема вследствие запрета на пересечения ветвей деревьев. Найдена главная поправка к координате ближайшей к нулю особой точки производяшей функции.Для рассмотренных простых гиперкубических решеток учтенной (отброшенной) петлей минимального размера является квадрат из четьрех двойных связей, образующийся при наложении двух узлов разных ветвей.

В данной работе тем же методом найдены главные поправки к координате особой точки при встраивании в большие деревья из двойных связей диаграмм (квадратов), образованных из шести связей (см. далее (9)), и диаграмм с четырехкратными взаимодейст- 
виями соседних спинов (см. (8)). Тем самым завершена начатая в [9] работа по определению координаты ближайшей к нулю особой точки спиновой коррелящионной функции методом разложения по $d^{-1}$. Полученный результат важен для высокотемпературной динамики спиновых систем, поскольку позволяет проследить изменение изучаемой характеристики при переходе от предельных к реальным величинам размерности пространства.

Во втором разделе статьи детально разобрано появление в процессе многократных коммутаций весового множителя у вкладов в спектральные моменты от интересующих нас простых решеточных фигур. В третьем разделе рассчитываются поправки к координате особой точки корреляционной функции. В п. 3.1 расчет выполнен при встраивании квадратов, в п. 3.2 - четырехкратных взаимодействий. В п. 3.3 найдена поправка к координате особой точки, связанная с уменьшением весового множителя базового дерева. В разделе 4 дано обсуждение полученных результатов.

В данной работе рассматриваются только системы с изотропным взаимодействием, хотя эксперименты выполнены как в системах с изотропным обменным взаимодействием [1]-[3], так и в системах с анизотропным диполь-дипольным взаимодействием [4]-[6]. При учете анизотропии теоретические формулы сильно усложняются [6], [8], [14], и этот случай составит предмет отдельного исследования.

\section{2. СПЕКТРАЛЬНЫЕ МОМЕНТЫ АВТОКОРРЕЛЯЦИОННОЙ ФУНКЦИИ}

Рассмотрим зависящую от времени автокорреляционную функцию спина, расположенного в одном из узлов простой гиперкубической решетки размерности $d$, при бесконечной температуре

$$
F(t)=\frac{\operatorname{Sp}\left\{e^{i \mathcal{H} t} S_{1}^{\alpha} e^{-i \mathcal{H} t} S_{1}^{\alpha}\right\}}{\operatorname{Sp}\left\{\left(S_{1}^{\alpha}\right)^{2}\right\}}
$$

где $\mathcal{H}$ - гамильтониан изотропной модели Гейзенберга

$$
\mathcal{H}=-\sum_{i, j} J_{i j}\left(S_{i}^{x} S_{j}^{x}+S_{i}^{y} S_{j}^{y}+S_{i}^{z} S_{j}^{z}\right)
$$

с взаимодействием $J$ между ближайшими $Z=2 d$ соседями, $S_{i}^{\alpha}-\alpha$-компонента $(\alpha=$ $x, y, z)$ векторного оператора спина в узле $i, S=1 / 2$. Эта функция может быть разложена в степенной ряд

$$
F(t)=\sum_{n=0}^{\infty} \frac{(-1)^{n}}{(2 n) !} M_{2 n} t^{2 n},
$$

где $n$-й коэффициент разложения определяется через $2 n$-кратный коммутатор

$$
M_{2 n}=\frac{\operatorname{Sp}\left\{\left[\mathcal{H},\left[\mathcal{H}, \ldots\left[\mathcal{H}, S_{1}^{\alpha}\right] \ldots\right]\right] S_{1}^{\alpha}\right\}}{\operatorname{Sp}\left\{\left(S_{1}^{\alpha}\right)^{2}\right\}}
$$

Как известно, $M_{2 n}$ является моментом порядка $2 n$ спектральной плотности автокорреляционной функции (1). 
Правила вычисления многократных коммутаторов в (4) подробно разбираются, например, в работах [7]-[11], [15], [18], [19]. При увеличении $n$ число слагаемых в моменте и сложность вычислений быстро возрастают, поэтому к настоящему времени рассчитаны моменты только до восьмого порядка [15]. Поскольку величина слагаемых зависит от выбора последовательности спиновых проекций и решеточных связей, Морита [15] предложил сгруппировать вклады по виду решеточных фигур из связей $(g)$,

$$
M_{2 n}=J^{2 n} \sum_{g} N(g) K_{2 n}(g),
$$

где $N(g)$ - число размешений без самопересечений данной фигуры на решетке, $J^{2 n} K_{2 n}(g)$ - вклад в момент, соответствуюший данной фигуре.

Будем использовать для наглядности изображения последовательности коммутаторов в (4) при нахождении $K_{2 n}(g)$ введенные в [7], [8] правила: вершина (точка на временной оси) - выполненная в соответствуюший момент времени операция коммутации; соединяюшая вершины линия с номером - оператор спиновой проекции соответствуюшего узла решетки. Второй момент

$$
M_{2}=2 J^{2} Z
$$

в изображении Мориты имеет вид

$$
1{ }^{\circ}{ }^{\circ}
$$

где цифры нумеруют узлы решеточной фигуры. В нашем изображении второй момент (6) есть

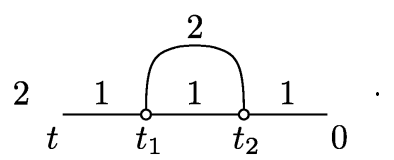

Весовой множитель 2 перед диаграммой обусловлен наличием двух вариантов выбора спиновых проекций дуги (спиновых проекций слагаемых гамильтониана (2) в коммутаторах по концам дуги). Общий множитель $J^{2 n}(5)$ перед каждой диаграммой не выписываем, а домножаем на него в конечном выражении.

Четвертый момент в нашем изображении представлен пятью вкладами
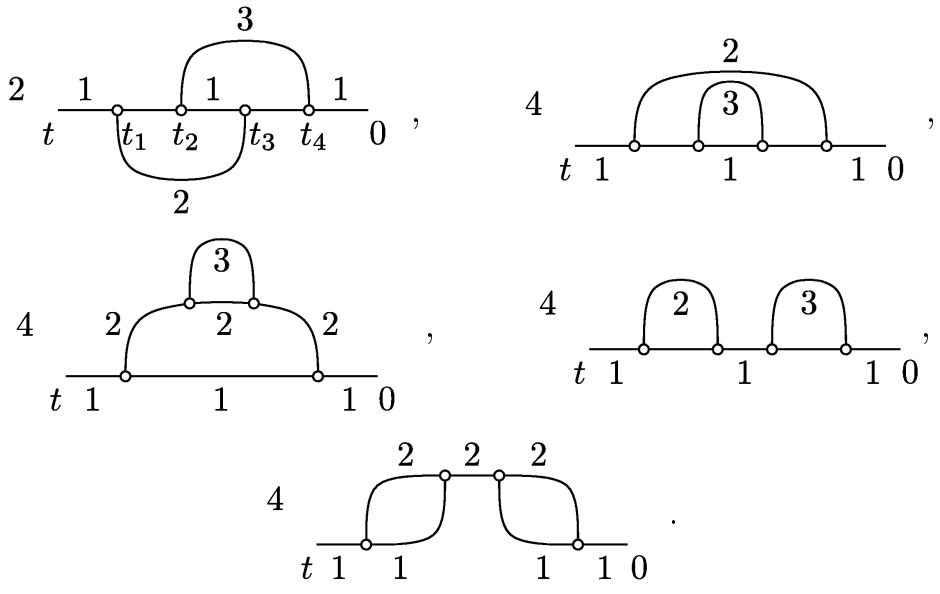
Весовой множитель перед каждой из диаграмм определяется числом вариантов выбора спиновых проекций на дугах. Его значение у первой диаграммы в (8) обусловлено требованием совпадения спиновых проекций двух дуг, необходимым для получения ненулевого вклада. Последняя диаграмма в (8) качественно отличается от предыдущих тем, что спиновой оператор сначала переходит с узла 1 на узел 2 , а потом возвращается, тогда как у остальных он остается все время на первом узле. Эта диаграмма представлена в обозначениях Мориты той же диаграммой (7), в которой подразумевается теперь не двукратное, а четырехкратное взаимодействие спинов 1 и 2. Сюда же попадает вклад от предпоследней диаграммы в (8) при совпадении номеров узлов двух дуг. При несовпадении узлов предпоследняя диаграмма и две первые дают вклад, представимый в обозначениях Мориты диаграммой

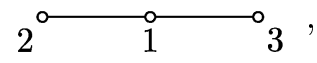

тогда как третья отличается положением первого спина в начале цепочки.

Вклады в моменты, представимые в изображении Мориты деревьями с максимальным числом узлов $\left(n+1\right.$ для $\left.M_{2 n}\right)$ подробно разобраны нами в работах [7]-[11]. Сейчас в качестве не сводимого к деревьям примера рассмотрим вклад в $M_{6}$, представленный диаграммой Мориты в виде квадрата из связей. Сушествует восемь способов построения квадрата с шестью связями-взаимодействиями:
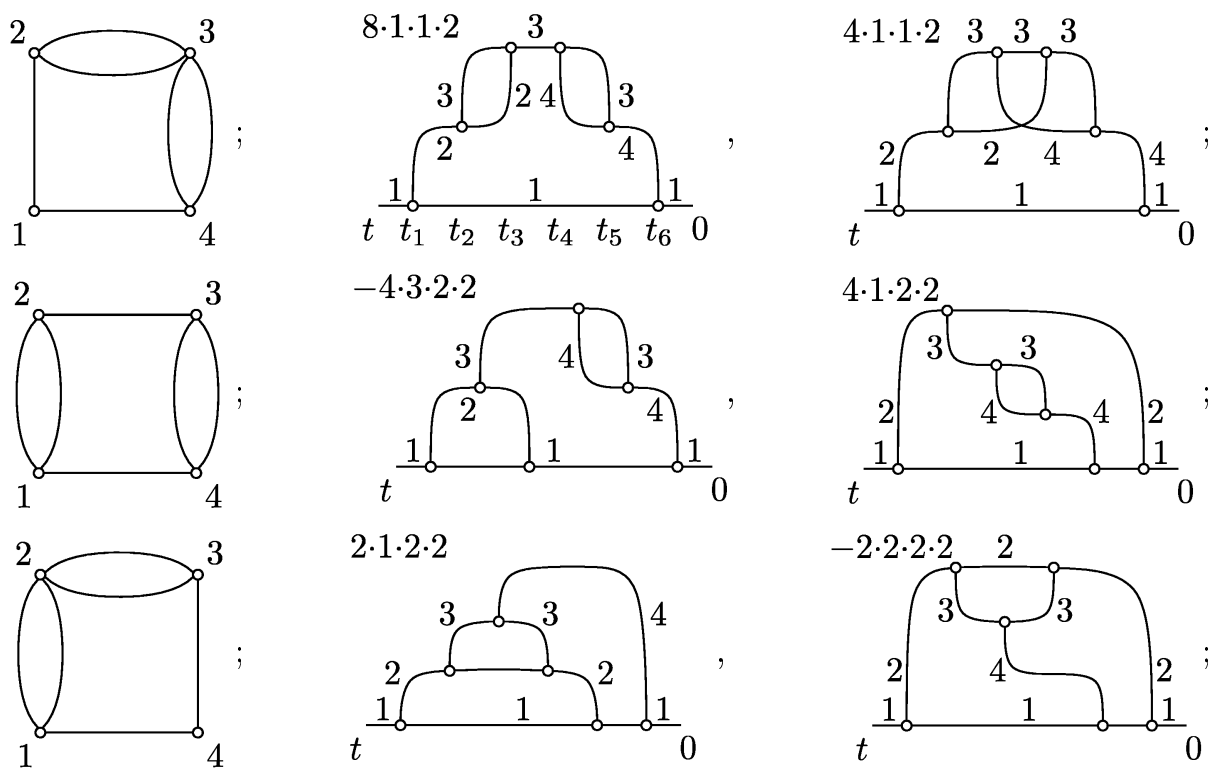

$$
-4 \cdot 3 \cdot 2 \cdot 2
$$
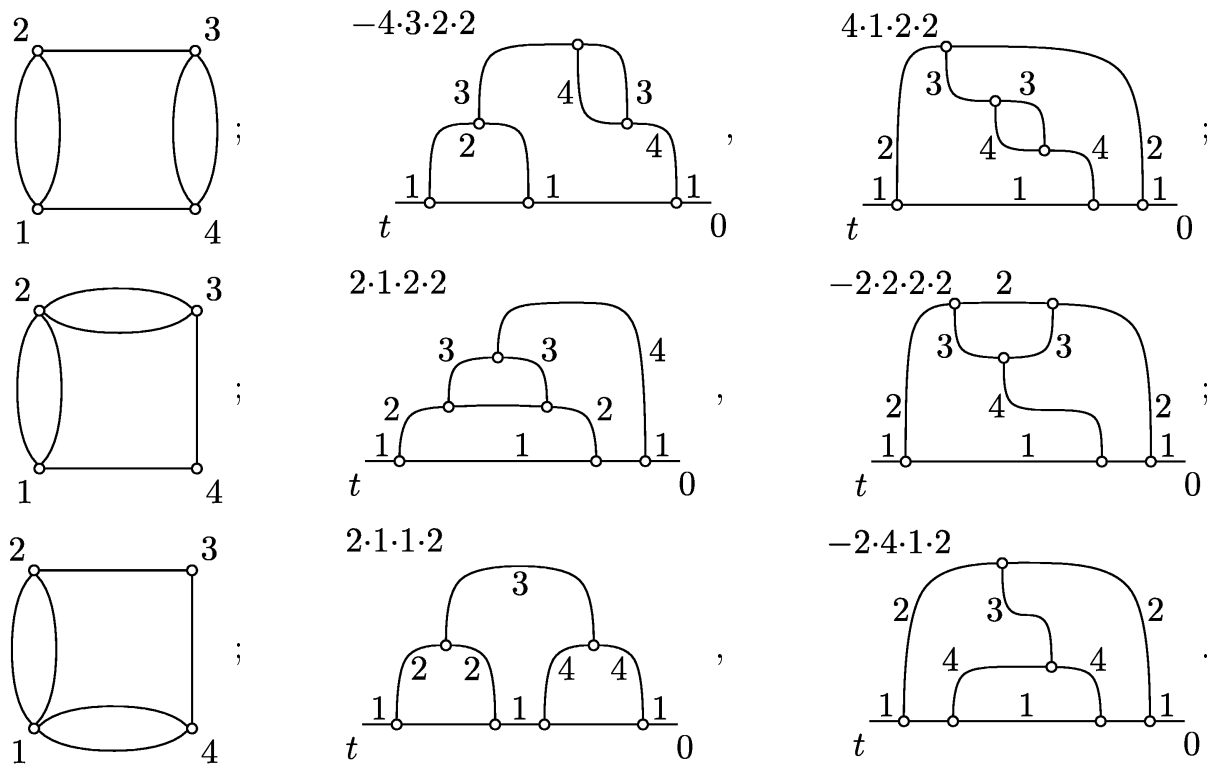
Здесь на диаграммах Мориты (слева) мы показали для наглядности кратность взаимодействий между спинами квадрата. В той же строке справа приведены наши диаграммы, поясняющие два варианта образования данной схемы связей при вычислении многократного коммутатора (4). Вклад каждой из диаграмм в момент является произведением четырех сомножителей: числа возможных вариантов выбора спиновых проекций дуг, числа взаимного расположения вершин на временной оси (отличного от единицы для вершин на разных линиях), коэффициента 2 для несимметричных диаграмм и общего множителя 2, связанного с перестановкой спинов 2 и 4 . Знак у каждого из вкладов получается как произведение знаков шести коммутаторов. Складывая все восемь вкладов, получаем - 28, что совпадает с результатом Мориты [15].

\section{3. РАСЧЕТ ПОПРАВОК К КООР ДИНАТЕ ОСОБОЙ ТОЧКИ КОРРЕЛЯЦИОННОЙ ФУНКЦИИ}

Достоинством приближения деревьев при независимом построении ветвей, использованным в работах [9]-[11], является возможность достаточно простого получения результатов для временной спиновой автокоррелящионной функции. Так, для координаты ее ближайшей особой точки на оси мнимого времени найдено значение [10]

$$
\tau_{b}=\tau_{\infty} \frac{\Gamma\left(2^{-1}-Z^{-1}\right)}{\sqrt{\pi} \Gamma\left(1-Z^{-1}\right)}
$$

где $\Gamma(x)$ - гамма-функция, $\tau_{\infty}=\pi /\left(2 M_{2}\right)^{1 / 2}$ - значение координаты в пределе $Z \rightarrow \infty$. Для реальных решеток можно пытаться найти необходимые поправки к точному решению упрощенной модели. Нас интересует характеристика объемных свойств кластеров: параметр роста их числа при увеличении числа связей или обратная ему величина координата особой точки производяшей функции. Поэтому главными будут поправки от петель малого размера, имеюших наибольший весовой множитель при их размешении на дереве. В работе [9] найдена поправка к координате особой точки вследствие исключения деревьев с пересечением ветвей в виде диаграммы (квадрата в представлении Мориты) из восьми связей. Сейчас в том же приближении рассчитаем поправки к координате особой точки (10) при учете встраивания в деревья диаграмм (квадратов) из шести связей (9), диаграмм четырехкратного взаимодействия соседних спинов (8), а также при учете уменьшения весового множителя дерева из-за свойств коммутаторов.

Поскольку особая точка расположена на оси мнимого времени, в формулах этого раздела перейдем к новой временной переменной

$$
\tau=t 2^{\frac{1}{2}} J i^{-1}
$$

где помимо мнимой единицы для упрошения формул введен масштабный множитель.

3.1. Поправка от квадрата. Когда квадрат (9) встроен в большое корневое дерево, то, во-первых, сушествует путь (один) к нему от корня, а во-вторых, сушествуют 
ветви, растушие из вершин квадрата. Согласно [9] соответствуюшую поправку к производяшей функции можно получить как решение уравнения

$$
\ddot{R}_{s}(\theta, \tau)=\ddot{f}_{s}(\tau)+\theta F_{k}(\tau) R_{s}(\theta, \tau)
$$

где $\theta$ служит параметром, помечающим число звеньев в цепи от корня к квадрату (в окончательных выражениях полагаем $\theta=Z-1)$. Функция

$$
F_{k}(\tau)=\left[F_{1}(\tau)\right]^{k}
$$

- это производящая функция $k=Z-2$ ветвей, растущих из узла цепи, $F_{1}(\tau)$ - производящая функция одной ветви (деревьев с висячим корнем), удовлетворяющая уравнению

$$
F_{1}(\tau)=1+\int_{0}^{\tau} d \tau_{1} \int_{0}^{\tau_{1}} d \tau_{2}\left[F_{1}\left(\tau_{2}\right)\right]^{\sigma}
$$

$f_{s}(\tau)$ - производящая функция деревьев, построенных на квадрате, присоединенном к корню одной связью, $\sigma=Z-1$.

Производящая функция $f_{s}(\tau)$ является суммой восьми вкладов, соответствующих восьми диаграммам (9),

$\ddot{f}_{s}(\tau)=F_{k-1}(\tau)\left[2 I_{1}(\tau)+I_{2}(\tau)-2 I_{3}(\tau)+2 I_{4}(\tau)+I_{5}(\tau)-I_{6}(\tau)+\frac{1}{2} I_{7}(\tau)-\frac{1}{2} I_{8}(\tau)\right]$.

Отличие коэффициентов перед интегралами в (15) от весовых множителей в (9) обусловлено двумя причинами. Во-первых, в (15) изменен масштаб времени (11), что приводит к уменьшению численного значения $M_{2 n}$ в $2^{n}$ раз. Во-вторых, множитель, обусловленный перестановкой вершин на временной оси, теперь учтен при записи пределов интегрирования (см. например, $I_{8}(\tau)$ ниже в формуле (16)).

Отдельный вклад для каждой диаграммы легко может быть записан по следуюшим правилам: каждой линии с узельным индексом 2,3 или 4 , начинаюшейся в вершине $t_{p}$ и оканчиваюшейся в вершине $t_{m}$, соответствует сомножитель $F_{k}\left(\tau_{p}-\tau_{m}\right)$ (с учетом $(11)$ ), узлу 1 соответствует сомножитель $F_{k-1}(\tau)$. По временным переменным вершин выполняется интегрирование. Пределы интегрирования последуюшей вершины определяются предыдущей вершиной на той же линии. Интегрирование по положению вершин на разных линиях выполняется независимо. В качестве примера приведем вклады первой и последней диаграмм в (9):

$$
\begin{aligned}
I_{1}(\tau)= & \int_{0}^{\tau} d \tau_{1} \int_{0}^{\tau_{1}} d \tau_{2} \int_{0}^{\tau_{2}} d \tau_{3} \int_{0}^{\tau_{3}} d \tau_{4} \int_{0}^{\tau_{4}} d \tau_{5} \int_{0}^{\tau_{5}} d \tau_{6} F_{k}\left(\tau_{1}-\tau_{3}\right) \times \\
& \times F_{k}\left(\tau_{2}-\tau_{5}\right) F_{k}\left(\tau_{4}-\tau_{6}\right), \\
I_{8}(\tau)= & \int_{0}^{\tau} d \tau_{1} \int_{0}^{\tau_{1}} d \tau_{6} \int_{\tau_{6}}^{\tau_{1}} d \tau_{3} \int_{\tau_{6}}^{\tau_{1}} d \tau_{2} \int_{\tau_{6}}^{\tau_{2}} d \tau_{5} \int_{\tau_{5}}^{\tau_{2}} d \tau_{4} F_{k}\left(\tau_{1}-\tau_{6}\right) \times \\
& \times F_{k}\left(\tau_{3}-\tau_{4}\right) F_{k}\left(\tau_{2}-\tau_{5}\right) .
\end{aligned}
$$

6 Теоретическая и математическая физика, т. 131, № 3, 2002 г. 
Координата особой точки определяется большими деревьями, которые генерируются производящей функцией при значении аргумента в окрестности особенности. Для главной части решения уравнения (12) в окрестности особой точки в статье [9] найдено значение

$$
R_{s}(\theta, \tau)=C_{s}\left(1-\frac{\tau}{\tau_{b}}\right)^{-1-\frac{2}{k}}
$$

где

$$
C_{s}=\frac{\tau_{b}}{3+\frac{4}{k}} \int_{0}^{\tau_{b}}\left(1-\frac{\tau}{\tau_{b}}\right)^{2+\frac{2}{k}} \ddot{f}_{s}(\tau) d \tau .
$$

Перейдя к переменной $\varphi=\pi \tau /\left(2 \tau_{b}\right)$, получаем

$$
C_{s}=\left(\frac{2}{\pi}\right)^{9} \frac{\tau_{b}^{8}}{3+\frac{4}{k}} \int_{0}^{\frac{\pi}{2}}\left(\frac{\pi}{2}-\varphi\right)^{2+\frac{2}{k}} \ddot{f}_{s}\left(\frac{2 \varphi \tau_{b}}{\pi}\right) d \varphi
$$

Вычислим коэффициент $C_{s}$ в интересуюшем нас пределе решеток большой размерности. При больших $Z$ порядок величины коэффициента перед интегралом в (19) согласно (10) равен $Z^{-4}$. Сам интеграл имеет порядок $Z^{0}$. Для нахождения его главной части в пределе $Z \rightarrow \infty$ в подьнтегральном выражении возьмем для функций $F_{k-1}(\tau)$ и $F_{k}(\tau)$ их предельную форму $F_{\infty}(\tau)=\cos ^{-2}\left(\pi \tau /\left(2 \tau_{b}\right)\right)$, а $(\pi / 2-\varphi)^{2+2 / k}$ заменим на $(\pi / 2-\varphi)^{2}$. Вычислив интеграл в $(19)$, получаем результат в виде восьми слагаемых по числу вкладов,

$$
2(0.016)+0.058-2(0.150)+2(0.171)+0.077-0.465+\frac{0.016}{2}-\frac{0.930}{2}=-0.715 .
$$

После подстановки в (19) этого значения и замены $\tau_{b}$ на $\tau_{\infty}=\pi /(2 Z)^{1 / 2}$ находим

$$
C_{s}=-0.715 \frac{2^{5}}{3 \pi Z^{4}}
$$

Далее, следуя [9], производящую функцию (17) деревьев со встроенными в них квадратами из связей-взаимодействий прибавим к производяшей функции деревьев $F_{1}(\tau)$ и будем трактовать эту добавку при $\tau \rightarrow \tau_{b}$ как первый член разложения $F_{1}(\tau)$ по $\delta \tau_{0 s}-$ поправке к координате особой точки. Отсюда получаем

$$
\delta \tau_{0 s}=-\frac{1}{2} C_{s} N_{4} \tau_{b} k
$$

где $N_{4}=k^{2} / 2$ - число размешений квадрата с закрепленной в данном узле одной из его вершин. Окончательно находим

$$
\delta \tau_{0 s}=0.607 \tau_{b} \sigma^{-1}
$$


3.2. Поправка от многократного взаимодействия. Для нахождения поправки от многократного взаимодействия между спинами в соседних узлах дерева рассмотрим две последние диаграммы (8) - простейшие диаграммы такого типа. При встраивании такого фрагмента в дерево ведущие к нему цепочки можно снова учесть уравнением (12) с другой функцией $f_{m}(\tau)$. Простейший вариант этой функции получается на основании этих диаграмм по тем же правилам, что и при записи (16). Однако при добавлении ветвей к узлам пары 1 и 2 возникает новое обстоятельство, связанное с исчезновением и появлением оператора в узлах 1 или 2. Поэтому оказывается возможным начать ветвь на одном временном интервале, а окончить на другом, как показано на диаграммах
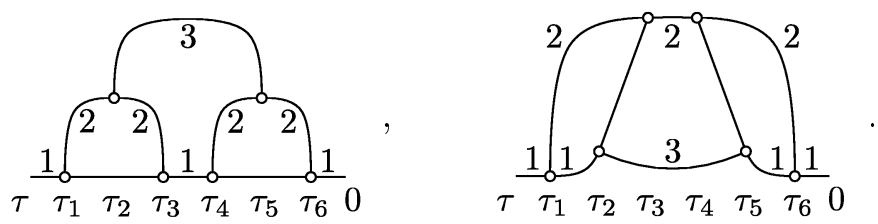

В первом случае в качестве спина 3 выступает один из $\sigma$ соседей спина 2 , а во втором из оставшихся $k$ соседей спина 1.

В том же приближении независимого построения ветвей деревьев эти ветви, построенные новым способом, легко объединить с прежними, взяв функцию $f_{m}(\tau)$ в виде суммы двух вкладов

$$
\begin{aligned}
& F_{k}(\tau)\left[F_{1}\left(\tau_{1}-\tau_{3}\right) F_{1}\left(\tau_{4}-\tau_{6}\right)+\int_{\tau_{3}}^{\tau_{1}} d \tau_{2} \int_{\tau_{6}}^{\tau_{4}} d \tau_{5} F_{\sigma}\left(\tau_{2}-\tau_{5}\right)\right]^{\sigma} \\
& F_{\sigma}\left(\tau_{1}-\tau_{6}\right)\left[F_{1}\left(\tau-\tau_{3}\right) F_{1}\left(\tau_{4}\right)+\int_{\tau_{3}}^{\tau} d \tau_{2} \int_{0}^{\tau_{4}} d \tau_{5} F_{\sigma}\left(\tau_{2}-\tau_{5}\right)\right]^{k}
\end{aligned}
$$

соответствующих двум вариантам взаимодействия спинов 1 и 2. Для получения окончательного выражения для $f_{m}(\tau)$ по оставшимся временным переменным вершин вьполняется интегрирование в пределах, легко выписываемых по виду двух диаграмм в (22).

После подстановки $f_{m}(\tau)$ в формулу $(18)$ вместо $f_{s}(\tau)$ и замены переменных получим по аналогии с (19)

$$
C_{m}=\left(\frac{2}{\pi}\right)^{7} \frac{\tau_{b}^{6}}{3+\frac{4}{k}} \int_{0}^{\frac{\pi}{2}}\left(\frac{\pi}{2}-\varphi\right)^{2+\frac{2}{k}} \ddot{f}_{m}\left(\frac{2 \varphi \tau_{b}}{\pi}\right) d \varphi .
$$

Перейдем к пределу $Z \rightarrow \infty$. Заменим в $(23)$ под интегралом функцию $F_{\sigma}(\tau)$ на $\cos ^{-2}\left(\pi \tau /\left(2 \tau_{b}\right)\right)$. После этого интеграл вычисляется и дает сумму четырех логарифмов. Первое слагаемое в каждой квадратной скобке в (23) согласно (14) дает единицу и еше два логарифма. В результате после замены в (23) степенной функции с большим показателем на экспоненциальную функцию получаем для первого слагаемого выражение

$$
\begin{aligned}
D_{1}(\varphi)= & \cos ^{-2}(\varphi) \int_{0}^{\varphi} d x_{1} \int_{0}^{x_{1}} d x_{2} \int_{0}^{x_{2}} d x_{3} \int_{0}^{x_{3}} d x_{4} \cos ^{2}\left(x_{1}-x_{3}\right) \cos ^{2}\left(x_{2}-x_{4}\right) \times \\
& \times \cos ^{-2}\left(x_{1}-x_{2}\right) \cos ^{-2}\left(x_{2}-x_{3}\right) \cos ^{-2}\left(x_{3}-x_{4}\right) \cos ^{-2}\left(x_{4}-x_{1}\right),
\end{aligned}
$$


а для второго -

$$
\begin{aligned}
D_{2}(\varphi)= & \cos ^{-2}(\varphi) \int_{0}^{\varphi} d x_{1} \int_{0}^{x_{1}} d x_{2} \int_{0}^{x_{2}} d x_{3} \int_{0}^{x_{3}} d x_{4} \cos ^{2}\left(\varphi-x_{3}\right) \cos ^{2}\left(x_{2}\right) \times \\
& \times \cos ^{-2}\left(\varphi-x_{2}\right) \cos ^{-2}\left(x_{2}-x_{3}\right) \cos ^{-2}\left(x_{3}\right) \cos ^{-2}\left(x_{4}-x_{1}\right) .
\end{aligned}
$$

После интегрирования по $\varphi$ получаем для двух соответствующих вкладов в (24) значения $D_{1}=0.174, D_{2}=0.136$, а для самого коэффициента -

$$
C_{m}=0.31 \frac{2^{4}}{3 \pi Z^{3}} .
$$

Для получения соответствующей поправки к координате особой точки согласно формуле (20) этот коэффициент следует домножить на число $N_{m}=\sigma$ положений многократной связи у узла и на $\tau_{b} k / 2$. Окончательно находим

$$
\delta \tau_{0 m}=-0.263 \frac{\tau_{b}}{\sigma} .
$$

Строго говоря, рассматриваемые выше поправочные члены, помимо главных - линейных по $1 / \sigma$ поправок, дадут вклады в поправки высших степеней. Мы их не учитываем ввиду относительной малости.

3.3. Поправка от уменьшения весового множителя дерева из-за свойств коммутаторов. Координата особой точки $\tau_{b}(10)$, входящая в формулы $(21)$ и (25), получена в приближении деревьев с максимальным комбинационным множителем. На примере множителя $M_{4}$ мы обнаруживаем его уменьшение от 4 до 2 для первой диаграммы (8). Соответствуюшую этой диаграмме поправку к производяшей функции получим описанным выше способом, взяв в $(18)$ вместо $f_{s}(\tau)$ функцию

$$
f_{c}(\tau)=-F_{k-1}(\tau) I_{c}(\tau)
$$

где

$$
I_{c}(\tau)=\int_{0}^{\tau} d \tau_{1} \int_{0}^{\tau_{1}} d \tau_{2} \int_{0}^{\tau_{2}} d \tau_{3} \int_{0}^{\tau_{3}} d \tau_{4} F_{\sigma}\left(\tau_{1}-\tau_{3}\right) F_{\sigma}\left(\tau_{2}-\tau_{4}\right)
$$

Оценим величину соответствуюшей поправки к координате особой точки в пределе $Z \rightarrow \infty$. Делая те же преобразования, что и в $(19)$ и $(24)$, и вычислив интеграл, находим

$$
C_{c}=-0.34 \frac{2^{4}}{3 \pi Z^{3}}
$$

После домножения согласно $(20)$ на $Z^{2} / 2$ (число способов размешений двух спинов данной диаграммы (8) у выделенного узла) и на $\tau_{b} k / 2$ получим

$$
\delta \tau_{0 c}=0.144 \tau_{\infty}
$$

Подобным же путем можно учесть поправки от множителей $M_{6}, M_{8}$ и т.д. Мы этого не делаем, поскольку ранее [7] по нелинейным уравнениям для автокорреляционной функции, выведенным с точностью до $M_{10}$, уже была найдена оценка координаты особой точки

$$
\tau_{0}=1.12 \tau_{\infty}
$$

После подстановки вместо $\tau_{\infty}$ в $(10)$ значения (28) мы получим интерполящионную формулу, позволяющую оценить влияние коммутационных поправок при уменьшении координационного числа в приближении Бете. 


\section{4. ОБСУЖДЕНИЕ}

Итак, методом разложения по обратной размерности пространства мы нашли главные поправки (21) и (25) к координате особой точки корреляционной функции от деревьев с встроенными фрагментами, содержащихся в точных выражениях для моментов. Ранее в работе [9] были найдены главные поправки в результате исключения деревьев с пересечениями ветвей. Собрав все эти поправки и учтя согласно (28) влияние коммутационных поправок, получаем окончательный результат для координаты на оси мнимого времени особой точки автокорреляционной функции спиновой системы с изотропным взаимодействием на простой гиперкубической решетке,

$$
\tau_{0} \approx 1.12 \tau_{b}\left(1+0.344 \sigma^{-1}+9.96 \sigma^{-2}\right) .
$$

Хотя два типа поправок оказались разного порядка по $\sigma^{-1}$, мы их привели вместе в окончательном выражении (29), поскольку они отражают качественно разные последовательности взаимодополняюших вкладов. Два подобных вклада были вычислены, например, для разветвленных полимеров ("lattice animals") [17], где в обоих случаях квадраты построены из четырех связей. Поэтому поправки имели один порядок величины по $\sigma^{-1}$, хотя различались численными коэффициентами и знаками. В нашем случае вследствие других свойств кластеров эти два вклада оказались разного порядка по $\sigma^{-1}$ и одного знака.

Полученный результат (29) позволяет проследить изменение координаты особой точки при уменьшении размерности пространства вплоть до $d=3$, поскольку поправки остаются еше достаточно малыми. В случае трехмерной решетки находим

$$
\tau_{0} \approx \frac{4.89}{\sqrt{M_{2}}} .
$$

По известному значению координаты особой точки корреляшионной функции можно получить оценку ее спектральных моментов высокого порядка, а также оценку далеких "крыльев" ее спектров. Наоборот, по измеренному далекому "крылу" спектра можно попытаться определить координату особой точки. Эксперименты [1]-[3], выполненные методами магнитного резонанса в системах с изотропным обменным взаимодействием, обнаружили экспоненциальное "крыло" спектра. К сожалению, в изученных системах на приводится значение обменного интеграла и, следовательно, множителя $M_{2}$ в формуле (30). Это не позволило нам сравнить теоретическое значение координаты особой точки с экспериментальным. Если же такое сравнение удастся выполнить в дальнейшем, то в зависимости от его результата будет подтверждена или опровергнута гипотеза об особой точке корреляционной функции. В свою очередь, этот выбор важен для понимания многочастичных эффектов в высокотемпературной динамике спиновых систем, для построения современной динамической теории и для интерпретации экспериментов.

Благодарности. В.Е. Зобов благодарит Б. Н. Провоторова, обратившего его внимание на близость проблемы оценки спектральных моментов высокого порядка к проблемам теории фазовых переходов. Работа выполнена при финансовой поддержке Российского фонда фундаментальных исследований (грант № 99-02-18214). 


\section{Список литературы}

[1] R. E. Walstedt. Phys. Rev. B. 1972. V. 5. P. 3782.

[2] W. G. Thomlinson, J. F. Kelly, R. C. Richardson. Phys. Lett. A. 1972. V. 38. P. 531.

[3] C. Cusumano, G. J. Troup. Phys. Stat. Sol. (b). 1974. V. 65. P. 655.

[4] D. A. McArthur, E. L. Hahn, R. E. Walstedt. Phys. Rev. 1969. V. 188. P. 609.

[5] H. T. Stokes, D. C. Ailion. Phys. Rev. B. 1977. V. 15. P. 1271.

[6] В. Е. Зобов, М. А. Попов, Ю.Н. Иванов, А. И. Ливииц. ЖЭТФ. 1999. Т. 115. С. 285. (Sov. Phys. JETP. 1999. V.88. P.157).

[7] В.Е. Зобов. ТМФ. 1988. Т. 77. С. 426.

[8] B. Е. Зобов. ТМФ. 1990. Т. 84. С. 111.

[9] В.Е. Зобов. ТМФ. 2000. Т. 123. С. 116.

[10] B. Е. Зобов, М. А. Попов. ТМФ. 1997. Т. 112. С. 479.

[11] V. E. Zobov, M. A. Popov. J. Stat. Phys. 1999. V. 97. P. 793.

[12] B. Е. Зобов, М. А. Попов. ТМФ. 2001. T. 126. С. 325.

[13] M. Blume, J. Hubbard. Phys. Rev. B. 1970. V. 1. P. 3815.

[14] В. Е. Зобов, А. А. Лундин. ЖЭТФ. 1994. Т. 106. С. 1097. (Sov. Phys. JETP. 1994. V.79. P. 595).

[15] T. Morita. J. Math. Phys. 1971. V. 12. P. 2062.

[16] M. E. Fisher, D.S. Gaunt. Phys. Rev. 1964. V. 133. P. A224.

[17] A. B. Harris. Phys. Rev. B. 1982. V. 26. P. 337.

[18] P. Resibois, M. De Leener. Phys. Rev. 1966. V. 152. P. 305.

[19] P. Borckmans, D. Walgraef. Physica. 1967. V. 35. P. 80; Phys. Rev. 1968. V. 167. P. 282.

Поступила в редакцию 23.VII.2001 г., после доработки 25.Х.2001 г. 